\title{
Calcium-Dependent Exocytosis of Atrial Natriuretic Peptide from Astrocytes
}

\author{
Mojca Krz̄an, ${ }^{3 \star}$ Matjaž Stenovec, ${ }^{2 \star}$ Marko Kreft, ${ }^{1,2}$ Tina Pangršič, ${ }^{2}$ Sonja Grilc, ${ }^{1}$ Philip G. Haydon, ${ }^{4}$ and Robert Zorec ${ }^{1,2}$ \\ ${ }^{1}$ Laboratory of Neuroendocrinology-Molecular Cell Physiology, Institute of Pathophysiology, Medical Faculty, University of Ljubljana, 1000 Ljubljana, \\ Slovenia, ${ }^{2}$ Celica Biomedical Sciences Center, 1000 Ljubljana, Slovenia, ${ }^{3}$ Institute of Pharmacology and Toxicology, Medical Faculty, University of Ljubljana, \\ Slovenia, and ${ }^{4}$ Department of Neuroscience, University of Pennsylvania School of Medicine, Philadelphia, Pennsylvania 19104
}

\begin{abstract}
Astrocytes are non-neuronal cells in the CNS, which, like neurons, are capable of releasing neuroactive molecules. However, the mechanism of release is ill defined. In this study, we investigated the mechanism of release of atrial natriuretic peptide (ANP) from cultured cortical astrocytes by confocal microscopy. To study the discharge of this hormone, we transfected astrocytes with a construct to express pro-ANP fused with the emerald green fluorescent protein (ANP.emd). The transfection of cells with ANP.emd resulted in fluorescent puncta in the cytoplasm that represent secretory organelles. If ANP is released by exocytosis, in which the vesicle fuses with the plasma membrane, then the total intensity of the green fluorescing probe should decrease, whereas the vesicle membrane is incorporated into the plasma membrane. To monitor exocytosis, we labeled the membrane with the fluorescent styryldye FM 4-64, a reporter of cumulative exocytosis. The application of ionomycin to elevate cytoplasmic $\left[\mathrm{Ca}^{2+}\right]$ increased the fluorescence intensity of FM 4-64, whereas that of ANP.emd decreased. These effects were not observed in the absence of extracellular $\mathrm{Ca}^{2+}$, suggesting that ANP is released by regulated $\mathrm{Ca}^{2+}$-dependent exocytosis from astrocytes.
\end{abstract}

Key words: astrocytes; glia; ANP; exocytosis; FM 4-64; fusion pore

\section{Introduction}

Astrocytes synthesize, store, and release many neuroactive compounds, including neurotransmitters (Parpura et al., 1994), neurotrophins, eicosanoids, and neuropeptides (Martin, 1992). A particular neuropeptide, atrial natriuretic peptide (ANP), has already been immunohistochemically identified not only in myocytes but also in neurons (McKenzie et al., 1990) and astrocytes (McKenzie, 1992) of canine and human cerebral cortex (McKenzie et al., 1994) and cerebellum (McKenzie et al., 2001). Myocardial cells store ANP in the form of prohormone in clathrin-coated vesicles (Klein et al., 1993) and release it by exocytosis when the prohormone is converted to the active form. The brain ANP is stored in vesicles and is released as a $24-25$ amino acid peptide. In the peripheral plasma, the 28 amino acid form of ANP predominates (Samson, 1987). Brain ANP content is significantly increased in astrocytes after experimental brain infarction (Nogami et al., 2001), suggesting that this putative neurotransmitter regulates cerebral blood flow. ANP is also involved in the control of salt appetite, because the destruction of ANP receptors eliminates the inhibition of salt appetite caused by an $\mathrm{NaCl}$ load (Blackburn et al., 1995).

Received Sept. 12, 2002; revised Dec. 10, 2002; accepted Dec. 12, 2002.

This work was supported by Ministry of Education, Sciences and Sports of The Republic of Slovenia Grant P3 521 0381, European Community Grant QLG3-CT 2001-2004, and Fogarty International Research Collaboration Award Grant R03-TW01293. We thank Dr. Ed Levitan for the generous donation of the ANP.emd construct.

${ }^{*}$ M.K. and M.S. contributed equally to this work.

Correspondence should be addressed to Robert Zorec, Laboratory of Neuroendocrinology-Molecular Cell Physiology, Institute of Pathophysiology, Medical School, University of Ljubljana, Zaloška 4, 1000 Ljubljana, Slovenia. E-mail: robert.zore@@mf.uni-lj.si.

Copyright $\odot 2003$ Society for Neuroscience $\quad 0270-6474 / 03 / 231580-04 \$ 15.00 / 0$
The mechanism of ANP release from astrocytes has not yet been determined. The release of atrial ANP from atrial myocytes was found to be triggered after muscarinic and vasopressin receptor activation (Sonnenberg and Veress, 1984) or after hemodynamic changes induced by isoproterenol and carbachol (Garcia et al., 1986), whereas for hypothalamic neurons, a depolarizationinduced, $\mathrm{Ca}^{2+}$-dependent mechanism of ANP release was determined (Tanaka and Inagami, 1986).

It is known that intracellular $\mathrm{Ca}^{2+}$ levels regulate the release of classical neurotransmitters, such as glutamate (Araque et al., 2000; Pasti et al., 2001) and aspartate. Astrocytes also possess several components of secretory apparatus, such as synaptobrevin II, cellubrevin, syntaxin (Parpura et al., 1995), and SNAP-23 (synaptosome-associated protein of $23 \mathrm{kDa}$ ) (Hepp et al., 1999); hence, ANP could be released via $\mathrm{Ca}^{2+}$-dependent exocytosis. To study the mechanism of ANP release from astrocytes, we transfected cells with a construct to express pro-ANP fused with the emerald green fluorescent protein (ANP.emd) (Han et al., 1999).

We show here that this construct labels discrete puncta in the cytoplasm that likely represent secretory organelles. Furthermore, we demonstrate that the application of ionomycin, in the presence, but not the absence, of extracellular calcium, increases the fluorescence intensity of FM 4-64, a fluorescent reporter of cumulative exocytosis, whereas the fluorescence intensity of ANP.emd-transfected cells decreases, suggesting that ANP is released via $\mathrm{Ca}^{2+}$-dependent exocytosis.

\section{Materials and Methods}

Astrocyte culture. Astrocyte cultures were prepared from the cortex of neonatal rats ( $3 \mathrm{~d}$ old $)$ and cultured as described previously (Schwartz and Wilson, 1992). Cells were grown in high glucose DMEM, containing $10 \%$ fetal 
bovine serum, $1 \mathrm{~mm}$ pyruvate, $2 \mathrm{~mm}$ glutamine, and $25 \mu \mathrm{g} / \mathrm{ml}$ penicillinstreptomycin in $95 \%$ air-5\% $\mathrm{CO}_{2}$. Confluent cultures were shaken at 225 rpm overnight, and the medium was changed the next morning; this was repeated for a total of three times. After the third overnight shaking, the cells were trypinsized and cultured for $24 \mathrm{hr}$ in $10 \mu \mathrm{M}$ cytosine arabinoside. After reaching confluence again, the cells were subcultured onto 22-mm-diameter circular poly-L-lysine-coated coverslips.

Lipofection. The plasmid encoding atrial natriuretic factor tagged with emerald green fluorescent protein, ANP.emd (a gift of Dr. Ed Levitan, University of Pittsburgh, Pittsburgh, PA) (Han et al., 1999), was introduced into the astrocytes by means of lipofection using the standard reference protocol of Invitrogen (Carlsbad, CA). DNA was mixed with 6 $\mu l$ of Plus Reagent (Invitrogen), diluted in $100 \mu$ l of serum-free DMEM, and incubated for $15 \mathrm{~min}$ at room temperature (RT). Lipofectamine (4 $\mu \mathrm{l}$; Invitrogen) was diluted in $100 \mu \mathrm{l}$ of serum-free DMEM. After incubation, both solutions were mixed and incubated again for $15 \mathrm{~min}$ at RT. In the meantime, astrocytes were washed once with serum-free DMEM and supplemented with $800 \mu \mathrm{l}$ of DMEM. The lipofection mixture (200 $\mu \mathrm{l}$ ) was pipetted onto the cells that were incubated for an additional $3 \mathrm{hr}$ at $95 \% \mathrm{O}_{2}-5 \% \mathrm{CO}_{2}$ at $37^{\circ} \mathrm{C}$. Then $30 \mu \mathrm{l}$ of Ultroser $\mathrm{G}$ (Invitrogen, Grand Island, NY) was added; DMEM was exchanged on the next day.

Confocal microscopy. Astrocyte-loaded coverslips were transferred into the recording chamber on a confocal microscope (model LSM 510; Zeiss, Jena, Germany) and supplied with $400 \mu \mathrm{l}$ of extracellular solution containing FM 4-64 (4 $\mu$ m; Molecular Probes, Leiden, The Netherlands). Cumulative exocytosis was monitored by measuring the intensity of FM 4-64 fluorescence (Betz et al., 1992; Smith and Betz, 1996; Kilic et al., 2001). To achieve this objective, cells were maintained in $4 \mu \mathrm{M}$ FM 4-64, which initially stains total plasma membrane. When vesicles subsequently fuse with the plasma membrane, the fluorescence intensity increases as FM 4-64 gains access to this newly exposed membrane. We report the FM 4-64 as a percentage change of the initial fluorescence before stimulus. A gravity-feed perfusion system (1-2 $\mathrm{ml} / \mathrm{min}$ ) was used to apply ionomycin $(10 \mu \mathrm{M})$ dissolved in extracellular solution containing FM 4-64. Fluorescent images were acquired by a plan-apochromatic oil immersion objective (63×, 1.4 numerical aperture) using $488 \mathrm{~nm}$ argon-ion laser excitation. ANP.emd and FM 4-64 fluorescence were separated using 505-530 $\mathrm{nm}$ bandpass and $580 \mathrm{~nm}$ long-pass emission filters, respectively. Images were stored on an IBM-PC compatible computer (Siemens, Nixdorf, Germany) and quantitatively analyzed using LSM 510 software (Zeiss) and a software subroutine written in MATLAB 5.3 (MathWorks, Natick, MA), using background subtraction. The number of points (vesicles) was determined using the Scion Image software (Scion, Frederick MD) and assuming that a point consists of at least five pixels, with a threshold of $25 \%$ of maximal fluorescence intensity.

Solutions. The extracellular solution contained the following (in mM): 130 $\mathrm{NaCl}, 5 \mathrm{KCl}, 8 \mathrm{CaCl}_{2}, 1 \mathrm{MgCl}_{2}, 10$ D-glucose, and $10 \mathrm{HEPES}, \mathrm{pH}$ adjusted to 7.2 using $\mathrm{NaOH}$. DMEM contained the following (in mM): 4 HEPES, 5 Tricine, $2 \mathrm{~L}$-glutamine, $10 \%$ newborn calf serum, and $0.01 \%$ bovine serum albumin. If not stated otherwise, all chemicals were obtained from Sigma (Darmstadt, Germany) and were of the highest purity grade.

Statistics. Statistical differences between FM 4-64 and ANP.emd fluorescence changes in $\mathrm{Ca}^{2+}$-free and $\mathrm{Ca}^{2+}$-containing solution were tested using the Student's $t$ test.

\section{Results}

Cultured cells were identified as astrocytes with immunocytochemical staining using antibodies against glial fibrillary acidic protein (data not shown), a molecule expressed only in differentiated astrocytes (Kennedy et al., 1980). The release of ANP from a single cell was studied by transfecting cells with a construct to express ANP.emd, which was shown previously to be targeted to secretory vesicles (Han et al., 1999). Figure 1 shows that, in transfected rat astrocytes, green fluorescence appears in puncta throughout the cytoplasm. These puncta represent ANPcontaining vesicles because immunocytochemical studies using an antibody against ANP and synaptobrevin II, a vesicle-
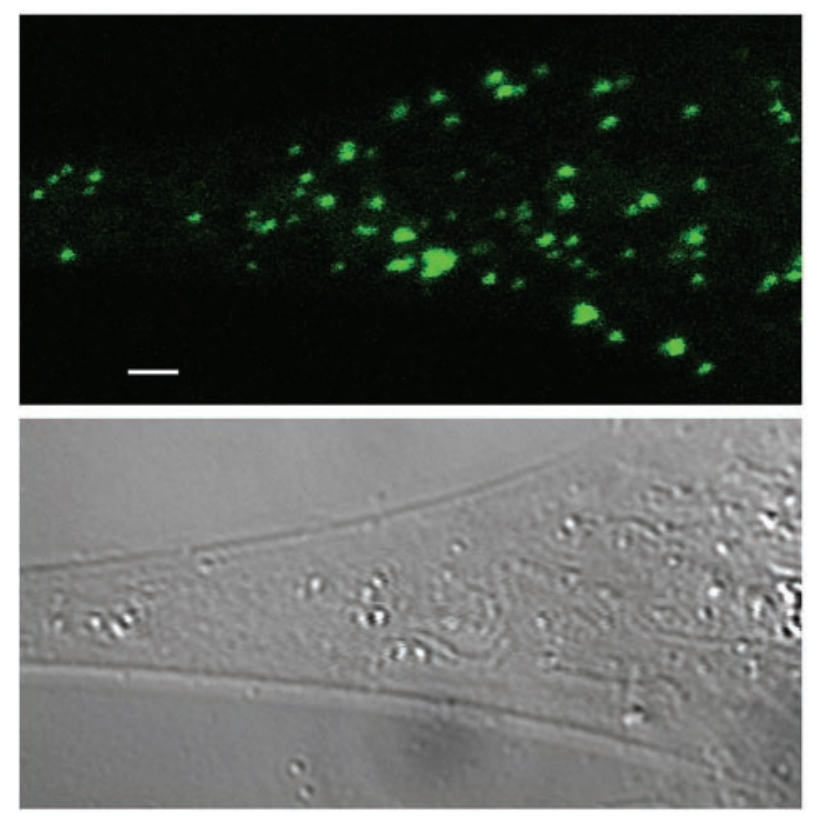

Figure 1. Confocal image of cultured cortical astrocyte transfected with an ANP.emd construct. Green fluorescent puncta are individual secretory granules that store ANP.emd (top). The same astrocyte is also observed under differential interference contrast optics (bottom). Scale bar, $2 \mu \mathrm{m}$.

associated protein, revealed colocalization of the green puncta with the signal from the ANP antibody and the synaptobrevin II antibody (data not shown). If ANP is released from astrocytes by regulated exocytosis, then the intensity of the green fluorescing probe should decrease (Han et al., 1999). Indeed, the stimulation of cells by the application of ionomycin to increase cytosolic $\left[\mathrm{Ca}^{2+}\right]$ resulted in an apparent decline in the number of green fluorescing puncta. We binarized the image shown in Figure 2 and counted the 381 puncta before and 203 puncta after the stimulus. The average intensity of pixels was similar: before ionomycin application, $60.0 \pm 0.7$ arbitrary units; after, $56.8 \pm 0.8$ arbitrary units (means $\pm \mathrm{SE}$ ). We also monitored timedependent changes in the fluorescence intensity by selecting a region of interest in the image of the whole cell. Immediately after the application of ionomycin, the average green fluorescence intensity increased transiently by $\sim 1 \%$, which is likely attributable to the neutralization of the vesicular $\mathrm{pH}$ through the open fusion pore (Barg et al., 2002) (see Discussion). Figures 3 and 4 show that, $1 \mathrm{~min}$ after the addition of ionomycin, the average green fluorescence intensity declined by $\sim 5 \%$. These results are consistent with the view that the decline in ANP.emd fluorescence is attributable to regulated exocytosis.

To test this hypothesis further, we monitored cumulative exocytosis by using the FM 4-64 styryl fluorescent probe, which stains membranes (Betz et al., 1992; Smith and Betz, 1996; Kilic et al., 2001). If the ionomycin-induced decline in green fluorescence intensity is attributable to regulated exocytosis, this should be associated with the incorporation of the new vesicle membrane into the plasma membrane (Smith and Betz, 1996); therefore, the intensity of FM 4-64 fluorescence should increase. In the presence of extracellular $\mathrm{Ca}^{2+}$, the FM 4-64 fluorescence intensity increased by $\sim 30 \% 1 \mathrm{~min}$ after ionomycin application (Figs. $3,4)$. The comparison of the latencies of the two fluorescence traces recorded in a single cell revealed no delay in the onset of the 
$0 \mathrm{~s}$
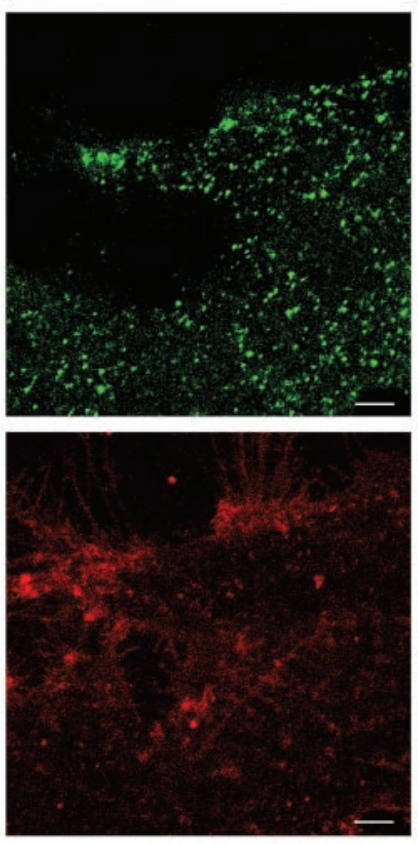

$60 \mathrm{~s}$
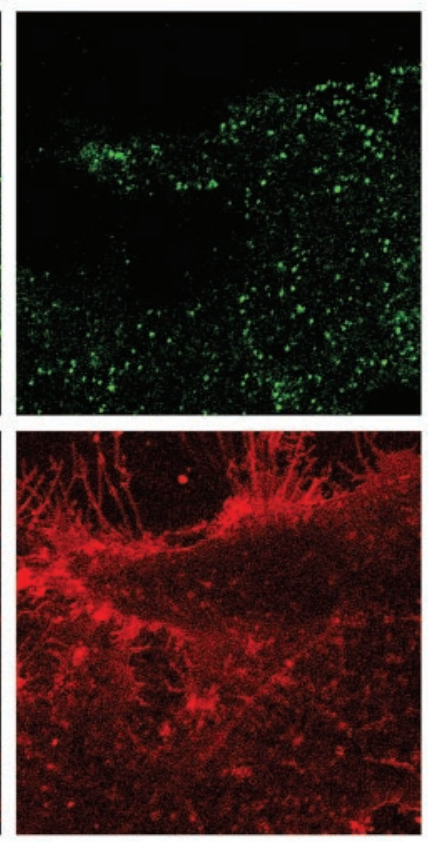

Figure 2. Ionomycin causes exocytotic release of ANP.emd. Confocal images of doublelabeled astrocyte before (left; 0 sec) and $60 \mathrm{sec}$ after (right) bath application of ionomycin (10 $\mu \mathrm{M})$ in the presence of $2 \mathrm{~mm} \mathrm{Ca}^{2+}$ in the bath solution. Top, ANP.emd fluorescence. Bottom, FM 4-64 stain. Note the decreased number of green fluorescent puncta and a large increase in the surface membrane area indicated by the intense FM 4-64 fluorescence staining after $60 \mathrm{sec}$. Scale bars, $5 \mu \mathrm{m}$.

FM 4-64 fluorescence intensity increase and the change in green fluorescence intensity (Fig. 3, inset). Both the ionomycininduced decline in the green fluorescence intensity and the ionomycin-induced increase in the red fluorescence intensity are consistent with an ionomycin-induced increased rate of exocytosis.

Regulated exocytosis requires a stimulus that results in an increase in cytoplasmic $\mathrm{Ca}^{2+}$ concentration (Burgoyne and Morgan, 1995; Calakos and Scheller, 1996). The application of the calcium ionophore ionomycin $(10 \mu \mathrm{M})$, which induces an increase in cytosolic $\left[\mathrm{Ca}^{2+}\right]$ in these cells (data not shown), induced changes in probe fluorescence intensity only in the presence of extracellular $\mathrm{Ca}^{2+}$ (Figs. 3, 4), which indicates that the release of ANP is mediated by regulated, $\mathrm{Ca}^{2+}$-dependent exocytosis.

\section{Discussion}

Although a large number of substances are reported to be secreted from astrocytes (Martin 1992, Parpura et al., 1994), there has been considerable debate about the underlying mechanisms. For example, in the case of the transmitter glutamate, the reversal of glutamate transporters, anion transporter-dependent mechanisms, and calcium-dependent exocytosis have all been proposed (Attwell, 1994). Although evidence is emerging to support the presence of an exocytotic mechanism, the demonstration of regulated exocytosis in these glial cells has remained elusive. The aim of this study was to determine the mechanism of ANP release from cultured rat cortical astrocytes and to thereby ask whether elevated internal calcium stimulates exocytosis in these nonneuronal cells. To this end, confocal microscopy was used. To

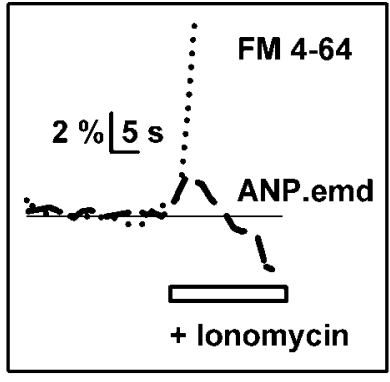

FM 4-64

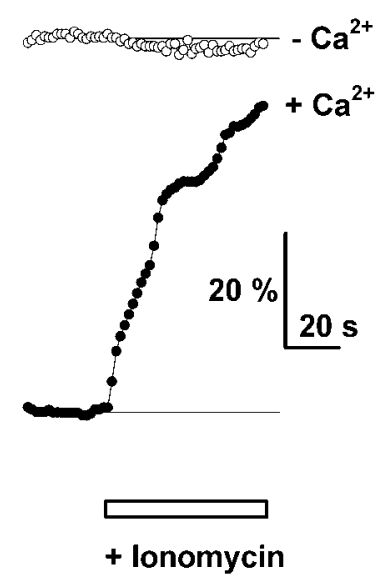

ANP.emd
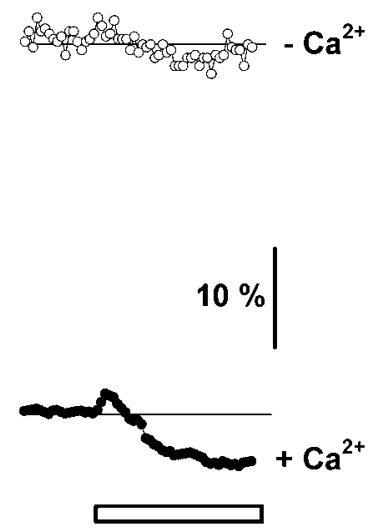

+ lonomycin
Figure 3. $\mathrm{Ca}^{2+}$-dependent release of ANP from cultured astrocytes. Normalized FM 4-64 fluorescence increase (left) and ANP.emd fluorescence decrease (right) obtained in a single astrocyte after the application of $10 \mu$ m ionomycin in $\mathrm{Ca}^{2+}$-free (top) and $\mathrm{Ca}^{2+}$-containing (2 $\mathrm{mm}$ ) extracellular solution (bottom). The inset shows the overlay of fluorescence changes obtained with ANP.emd (filled symbols) and FM 4-64 (open symbols), showing no delay in the onset of ionomycin-induced fluorescence changes.
FM4-64

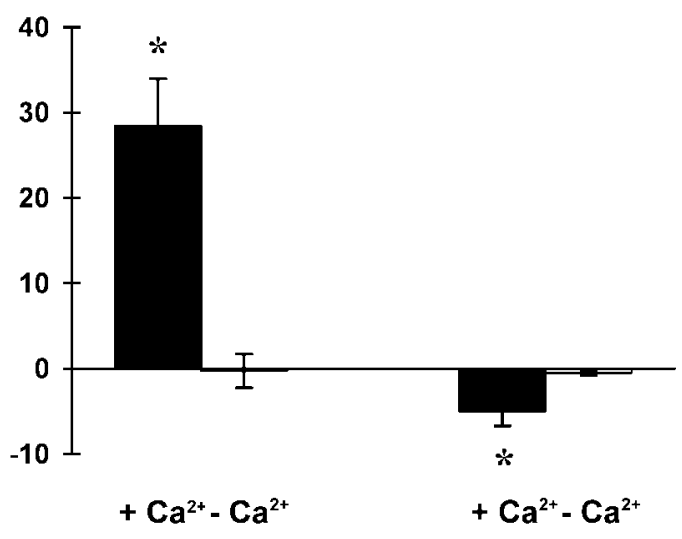

Figure 4. Mean relative change of FM 4-64 (left) and ANP.emd (right) fluorescence obtained in different cells stimulated with $10 \mu \mathrm{m}$ ionomycin in extracellular solution containing $2 \mathrm{~mm}$ $\mathrm{Ca}^{2+}(n=7)$ and $\mathrm{Ca}^{2+}$-free extracellular solution $(n=5)$. Asterisks indicate a statistically significant difference in the change in FM 4-64 and ANP.emd fluorescence change in the presence or absence of extracellular C ${ }^{2+}$ (FM 4-64, $p=0.0018$; ANP.emd, $p=0.036 ; t$ test).

monitor the release of the neuropeptide, we transfected cells with a construct to express ANP.emd (Han et al., 1999). In our successfully transfected cells, fluorescence appeared as discrete puncta. 


\section{Calcium-dependent exocytosis}

Consistent with the presence of a regulated exocytosis pathway, the addition of the $\mathrm{Ca}^{2+}$ ionophore ionomycin stimulated a reduction in the average fluorescent intensity of the cell that resulted from a reduction in the number of ANP.emd puncta within the astrocyte. Because the omission of $\mathrm{Ca}^{2+}$ from the bathing medium prevented the ionophore-induced release of ANP.emd, these data are consistent with the $\mathrm{Ca}^{2+}$-dependent exocyotsis of peptide from astrocytes.

To provide an alternative approach to study exocytosis, we asked whether the ionophore-induced release of ANP caused a correlated increase in membrane surface area that would support the presence of exocytotic release of this peptide. Using FM 4-64 to monitor cumulative exocytosis (Betz et al., 1992; Smith and Betz, 1996; Kilic et al., 2001), we found a $\mathrm{Ca}^{2+}$-dependent increase in total FM 4-64 fluorescence intensity.

\section{Fusion pore dilation}

When the kinetics of cumulative exocytosis (FM 4-64) are superimposed on the fluorescence of ANP.emd, no delay was observed between the stimulus and the increase of FM 4-64 intensity. Immediately after ionomycin application, the intensity of ANP.emd transiently increased. This increase in intensity results from the $\mathrm{pH}$ sensitivity of ANP.emd (Barg et al., 2002). Before the opening of the fusion pore, vesicles are acidic, which reduces the intensity of emd fluorescence. Immediately after the stimulus, the initial opening of the fusion pore allowed protons to leave the vesicle, which led to a dequenching and thus to an increase in ANP.emd fluorescence (Barg et al., 2002). It is likely that the diameter of this initial fusion pore is sufficiently large to permeate not only protons but also the lowmolecular-weight dye FM 4-64. After the initial rise in green fluorescence, the intensity of ANP.emd decreased, which we interpret as being attributable to the ANP.emd release into the bathing saline. Regardless of the details of the kinetics of these fluorescence changes, when taken together, these data show that the application of ionomycin to elevate astrocytic internal $\left[\mathrm{Ca}^{2+}\right]$ stimulated the release of ANP.emd through an exocytotic mechanism.

This clear demonstration of the regulated exocytotic release of ANP from astrocytes has two important outcomes for our understanding of the physiology of astrocytes. First, the demonstration of $\mathrm{Ca}^{2+}$-regulated exocyotsis in these non-neuronal cells indicates that, other chemical transmitters released from these cells, such as glutamate, have the potential to be released by an exocytotic mechanism. Second, the demonstration of $\mathrm{Ca}^{2+}$-dependent ANP release from astrocytes raises the hypothesis that synaptic activity might regulate cerebral blood flow via the release of ANP from astrocytes. According to this notion, synaptic activity, which is known to be able to induce $\mathrm{Ca}^{2+}$ signaling in astrocytes, could cause the release of endogenous ANP from astrocytes to regulate the cerebral microvessels. In agreement with this possibility, ANP has been demonstrated to be present in astrocytes, and it has been suggested that it may regulate cerebral blood flow (McKenzie et al., 2001), because this peptide is known to cause vasodilation (Kubo et al., 1992).

In summary, these studies demonstrate that an increase in the cytoplasmic $\left[\mathrm{Ca}^{2+}\right]$ causes the exocytotic release of ANP from astrocytes. The demonstrated release of ANP from astrocytes by an exocytotic pathway suggests that astrocytes actively regulate their local neuronal, synaptic, and endothelial environment by the release of a variety of chemical transmitters.

\section{References}

Araque A, Li N, Doyle RT, Haydon PG (2000) SNARE-protein-dependent glutamate release from astrocytes. J Neurosci 20:666-673.
Attwell D (1994) Glia and neurons in dialogue. Nature 369:707-708.

Barg S, Olofsson CS, Schriever-Aeln J, Wendt A, Gebre-Medhin S, Renstrom E, Rorsman P (2002) Delay between fusion pore opening and peptide release from large dense-core vesicles in neuroendocrine cells. Neuron 33:287-299.

Betz WJ, Mao F, Smith CB (1992) Activity-dependent fluorescent staining and destaining of living vertebrate motor nerve terminals. J Neurosci 12:363-375.

Blackburn RE, Samson WK, Fulton RJ, Stricker EM, Verbalis JG (1995) Central oxytocin and ANP receptors mediate osmotic inhibition of salt appetite in rats. Am J Physiol 269:R245-R251.

Burgoyne BD, Morgan A (1995) $\mathrm{Ca}^{2+}$ and secretory dynamics. Trends Neurosci 18:191-196.

Calakos N, Scheller RH (1996) Synaptic vesicle biogenesis, docking, and fusion: molecular description. Physiol Rev 76:1-29.

Garcia R, Lacance D, Thibault G, Cantin M, Gutkowska J (1986) Mechanisms of release of atrial natriuretic factor. II. Effect of chronic administration of $\alpha$ - and $\beta$-adrenergic and cholinergic agonists on plasma and atrial ANF in the rat. Biochem Biophys Res Commun 136:510-520.

Han W, Ng YK, Axelrod D, Levitan ES (1999) Neuropeptide release by efficient recruitment of diffusing cytoplasmic secretory vesicles. Proc Natl Acad Sci USA 96:14577-14582.

Hepp R, Perraut M, Chasserot-Golaz S, Galli T, Aunis D, Langley K, Grant NJ (1999) Cultured glial cells express the SNAP-25 analogue SNAP-23. Glia 27:181-187.

Kennedy PG, Lisak RP, Raff MC (1980) Cell type-specific markers for human glial and neuronal cells in culture. Lab Invest 43:342-351.

Kilic G, Angelson JK, Cochilla AJ, Nussinovitch I, Betz WJ (2001) Sustained stimulation of exocytosis triggers continuous membrane retrieval in rat pituitary somatotrophs. J Physiol (Lond) 532:771-783.

Klein RM, Kelley KB, Merisko-Liversidge EM (1993) A Clathrin-coated vesicle-mediated pathway in atrial natriuretic peptide (ANP) secretion. J Mol Cell Cardiol 25:437-452.

Kubo SH, Atlas SA, Laragh JH, Cody RJ (1992) Maintenance of forearm vasodilator action of atrial natriuretic factor in congestive heart failure secondary to ischemic or idiopathic dilated cardiomyopathy. Am J Cardiol 69:1306-1309.

Martin DL (1992) Synthesis and release of neuroactive substances by glia. Glia 5:81-94

McKenzie JC (1992) Atrial natriuretic peptide-like immunoreactivity in astrocytes of parenchyma and glia limitans of the canine brain. J Histochem Cytochem 40:1211-1222.

McKenzie JC, Cowie RJ, Inagami T (1990) ANP-like immunoreactivity in neuronal perikarya and processes associated with vessels of the pia and cerebral parenchyma in dog. Neurosci Lett 117:253-258.

McKenzie JC, Berman NEJ, Thomas CR, Young JK, Compton LY, Cothran LN, Liu W-L, Klein RM (1994) Atrial natriuretic peptide-like immunoreactive (ANP-LIR) neurons and astroglia of the human cerebral cortex. Glia 12:228-243.

McKenzie JC, Juan YW, Thomas CR, Berman NE, Klein RM (2001) Atrial natriuretic peptide-like immunoreactivity in neurons and astrocytes of human cerebellum and inferior olivary complex. J Histochem Cytochem 49:1453-1467.

Nogami M, Shiga J, Takatsu A, Endo N, Ishiyama I (2001) Immunohistochemistry of atrial natriuretic peptide in brain infarction. Histochem J 33:87-90.

Parpura V, Basarky TA, Liu F, Jeftinija K, Jeftinija S, Haydon PG (1994) Glutamate-mediated astrocyte-neuronal signalling. Nature 369:744-747.

Parpura V, Fang Y, Basarsky T, Jahn R, Haydon PG (1995) Expression of synaptobrevin II, cellubrevin and syntaxin but not SNAP-25 in cultured astrocytes. FEBS Lett 377:489-492.

Pasti L, Zonta M, Pozzan T, Vicini S, Carmignoto G (2001) Cytosolic calcium oscillations in astrocytes may regulate exocytotic release of glutamate. J Neurosci 21:477-484.

Samson WK (1987) Atrial natriuretic factor and the central nervous system. Endocrinol Metab Clin North Am 16:145-161.

Schwartz JP, Wilson DJ (1992) Preparation and characterization of type 1 astrocytes cultured from adult rat cortex, cerebellum and striatum. Glia 5:75-80.

Smith CB, Betz WJ (1996) Simultaneous independent measurement of endocytosis and exocytosis. Nature 380:531-534.

Sonnenberg H, Veress AT (1984) Cellular mechanism of release of atrial natriuretic factor. Biochem Biophys Res Commun 124:443-449.

Tanaka I, Inagami T (1986) Release of immunoactive atrial natriuretic factor from rat hypothalamus in vitro 122:353-355. 